\title{
ARTICLE
}

Cite this: DOI: $10.1039 / \times 0 \times x 00000 x$

\section{Droplet-based electro-coalescence for probing threshold disjoining pressure}

\author{
Zhou Liu, ${ }^{\text {ab }}$ San To Chan ${ }^{\text {a }}$, Faizi Hammad Ali ${ }^{\text {a }}$, Robert C. Roberts ${ }^{c}$ and Ho Cheung \\ Shum ${ }^{* a b}$
}

Received 00th January 2012,

Accepted 00th January 2012

DOI: $10.1039 / \times 0 \times x 00000 x$

www.rsc.org/
In this work, we investigate the coalescence of emulsion droplets in a controlled electric field. Two contacting droplets stabilized by surfactants can be forced to coalesce into a combined one when the applied voltage is above a critical value. The critical voltages change with the types, concentrations of surfactants and temperature. By exploring the drainage of thin oil film trapped between emulsions, we interpret that the coalescence occurs as the electric compression overcomes the disjoining pressure barrier and squeezes the film to a critical thickness. Based on this, we have devised an approach to probe the threshold disjoining pressure which can help predict the emulsion stability and surfactant efficacy quantitatively. We have confirmed the validity of our approach for measuring the threshold disjoining pressure by comparing the result with other proven tests that involve centrifugation and thermal heating. Our approach is simple, reliable and robust in predicting emulsion stability, and will facilitate the design of emulsion-based formulations by accelerating the testing of emulsion stability.

\section{Introduction}

Emulsions, mixtures of two immiscible fluids with one phase dispersed in another, have various applications; these include food products, cosmetics, pharmaceutics ${ }^{1-3}$, as well as droplet-based microfluidic sensors ${ }^{4-6}$ and biological assays ${ }^{7-10}$. The shelf life of the emulsion products is mainly determined by the emulsion stability, which represent their capability to resist changes in properties over time. $^{2}$ To enhance the emulsion stability, surfactants are usually added to prevent the coalescence of droplets. These surfactant molecules aggregate at interfaces and provide a repulsive stress Prepulsion, which prevents the direct contact of the emulsions in close proximity. ${ }^{11}$ The repulsive stress can be either long-ranged or short-ranged. An example of long-ranged interactions is electrostatic interactions provided by ionic surfactants. ${ }^{12}$ A common short-ranged interaction is steric stress caused by the overlapping of polymeric chains of non-ionic surfactants. ${ }^{12}$ Sources of interactions counteracting these repulsions include the attractive van der Waals stress $P_{\mathrm{vdw}}=-\mathrm{A} / 6 \pi h^{3}$, which tends to facilitate the approach of the interfaces, ${ }^{13-15}$ where $\mathrm{A}$ is the Hamaker constant and $h$ is the thickness of the oil film. The net interaction between the interfaces, which is the sum of the repulsive stress and attractive stress: $\Pi(h)=P_{\text {repulsion }}+P_{\mathrm{vdw}}$, is often called a disjoining pressure. ${ }^{11,16,17}$ Interestingly, the disjoining pressure is not a function of the film thickness $\mathrm{h}$ monotonically. ${ }^{18}$ With a decrease in film thickness, the disjoining pressure rises as the repulsive stress dominates the interaction of interfaces initially. At sufficiently small film thickness, the pressure reaches a peak and decreases as the attractive stress dominates the interaction of the interfaces. Thus, the disjoining pressure isotherm contains a metastable point, sometimes termed the threshold disjoining pressure $\prod_{\text {threshold }}$, which represents physical pressure barrier that determines the stability of emulsions.
Knowledge of the threshold disjoining pressure $\prod_{\text {threshold }}$ can help to predict the emulsion stability, which is especially crucial to the design of emulsion-based formulations ${ }^{2}$ and the manipulation of droplets in emulsion--based techniques ${ }^{19-23}$. Traditional method to measure the disjoining pressure isotherm usually utilizes the porousplate technique, ${ }^{24}$ which probes the $\Pi(h)$ through hydrostatic pressure when the film is in an equilibrium state. However, the threshold value of the disjoining pressure is much more rarely measured. This may be due to the limited pressure range of porousplate technique as measuring $\prod_{\text {threshold }}$ usually involves a process of breaking the film under relatively large external pressure. There are also alternative measurement techniques utilizing external pressure or thermal heating to probe $\prod_{\text {threshold }}$, but most of these approaches have limitations and lack sound scientific basis. ${ }^{2,25,26}$ For instance, centrifugation approach can break the emulsion droplets and determines the $\prod_{\text {threshold }}$ through an external centrifugal force. This method is ineffective when applied to emulsions without distinct density difference between the two emulsion phases.

In this work, we use a custom-built system to study the coalescence of emulsion droplets under a controlled electric field. Two emulsion droplets upon contact can be prevented from coalescing using surfactants. An applied voltage can facilitate the coalescence of these surfactant-stabilized droplets ${ }^{27-29}$. From our studies, emulsion droplets always coalesce when the voltage is above a critical value; this critical voltage differs with the types, concentrations of surfactants and even temperature. Based on framework of disjoining pressure, we attribute the coalescence of droplets to the electric compression which overcomes the disjoining pressure barrier when the applied voltage is sufficiently large. With these understandings, we further devise an approach to deduce the $\prod_{\text {threshold }}$ of emulsions stabilized by different surfactants. The result of $\prod_{\text {threshold }}$ is in good agreement with that tested by other proven methods. Our work suggests that the $\prod_{\text {threshold }}$ determined by droplet- 
based electro-coalescence has great potential as a general quantitative indicator for emulsion stability. By inducing droplets to coalescence within timescales much shorter than that between uncharged droplets, our approach accelerates the testing of emulsion stability and will contribute to the design of emulsion-based formulations.

\section{Experimental}

\section{Emulsion preparation}

We form a pair of water droplets submerged in oil continuous phase in a custom-built poly (methyl methacrylate) (PMMA) device, as shown schematically in Fig 1(a). Deionized water with different concentrations of potassium chloride $(\mathrm{KCl})$ is used as the dispersed phase. The ionic strengths of these aqueous phases are measured using an electric conductivity meter (Eutech Inc., CONT 610). Silicone oil (10mPa $\cdot s$, Aladdin Reagent) or liquid paraffin (36.5110 $\mathrm{mPa} \cdot \mathrm{s}$, Aladdin Reagent) is used as the continuous phase. To form two contacting emulsion droplets, we slowly inject the aqueous phase into the oil phase through two opposing needles using syringe pumps (Longer pumps, Baoding, China). Prior to contact, we stop the injection of fluids and let the droplets gradually get into contact. The coalescence of emulsion droplets is prevented by stabilizers that include Span 80 (Sigma, USA), EM90 (ABIL, USA), Dow Corning 749 (Dow Corning, USA), as shown in Fig 1(b). The corresponding interfacial tension between the dispersed phase and continuous phase is measured using a spinning drop tensiometer (Kruss-SITE100).

\section{Electro-coalescence and temperature control}

After the formation of the two touching droplets, we use an electrochemical workstation (Model: $\mathrm{CH}$ Instruments, 660E) to apply a direct current (DC) and monitor the corresponding current. The voltage gets ramped up linearly with controlled scanning rate. The corresponding dynamic behaviours of the emulsion droplet pair are monitored under an optical microscope (Motic AE2000) connected to a high-speed camera (Phantom v9.1) with frame rates of up to $2000 \mathrm{f} \cdot \mathrm{s}^{-1}$. To investigate the critical voltages at different temperatures, we control the temperature of the liquid using a heating plate (Bioscience Tools) connected to the microscope stage. The temperature of liquid after heating is monitored using an infrared thermometer (Smart sensor, AR350) during the electrocoalescence experiments.

\section{Measurement of film thickness}

To measure the film thickness, we superimpose a sinusoidal waveform onto the base DC voltage. The influence of sinusoidal waveform on the film thickness can be neglected due to its low amplitude compared with the base DC voltage. Afterwards, we record the behaviours of the droplets and monitor their corresponding current signal. The impedance $Z$ can be calculated by dividing the applied voltage over current signal. Considering oil is dielectric, we treat the thin film as a parallel-plate capacitor with a capacitance: $C=\varepsilon_{\mathrm{r}} \varepsilon_{0} S / h$, where $\varepsilon_{0}$ is the vacuum permittivity, $\varepsilon_{\mathrm{r}}$ is the dielectric constant of oil, $S$ is the contact area (measured by microscopic images) and $\mathrm{h}$ is the film thickness. The capacitance of the thin film contributes the most to the imaginary part of the impedance: $Z^{\prime \prime}=1 / j \omega C$, where the $j$ is the imaginary unit, $\omega$ is the frequency. Thus, we can calculate the film thickness using the following formula: $h=\varepsilon_{\mathrm{r}} \varepsilon_{0} \cdot S \cdot \omega \cdot\left|Z^{\prime \prime}\right|$.

\section{Centrifuge acceleration and thermal heating}

To test the emulsion stability using an accelerated test based on centrifugation, ${ }^{25}$ we first disperse water droplets in paraffin oil. These water droplets are stabilized by the surfactants: $2 \%$ EM 90 or $2 \%$ Span 80 . For all emulsion samples, the volume of water and oil is kept the same to ensure a fair comparison. ${ }^{25}$ The emulsions are placed in centrifuge tubes and rotated by a centrifuge at $1000 \mathrm{rpm}$ or $2000 \mathrm{rpm}$ for 3 minutes. Immediately after centrifugation, we retrieve the emulsion and collect relevant images for analysis. To test the emulsion stability using thermal heating method, we first fabricate water-in-oil emulsions stabilized by $2 \%$ EM90 or $2 \%$ Span 80 using a capillary microfluidic device. ${ }^{30}$ The resultant emulsion samples are uniform in droplet size. Afterwards, we place these emulsion samples into an oven at a constant temperature of $50^{\circ} \mathrm{C}$; the droplet size is continuously monitored for characterization of the emulsion stability.

(a)

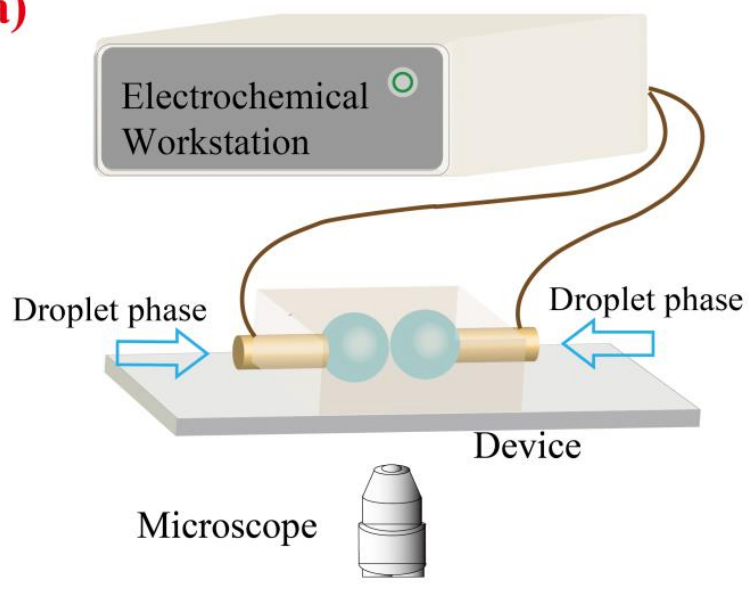

(b)

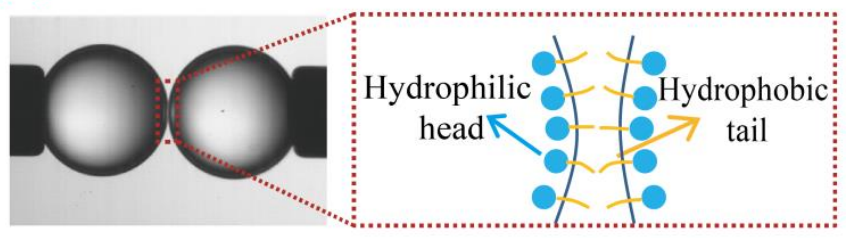

Fig. 1 (a) Schematic of droplet-based electro-coalescence. Two contacting emulsion droplets are placed under an electric field controlled by an electrochemical workstation. (b) Microscopic image of two emulsions stabilized by surfactants. Surfactants absorbed on water/oil interfaces prevent the direct contact of emulsions as sketched by the insert.

\section{Results and discussion}

\section{Electro-coalescence of emulsion droplets}

To investigate the coalescence of emulsions in an electric field, we charge the two droplets oppositely by applying a voltage through the two metallic nozzles. We increase the applied DC voltage linearly with a constant scanning rate, as indicated by the red dots in Fig 2(a). At nil or low applied voltage, the emulsion pair remains stable (inserts: $0 \mathrm{~V} \sim 1.0 \mathrm{~V}$, Fig 2(a)). The stable state of droplets is also reflected by the nil current in the initial current evolution (green dots, Fig 2(a)). When the applied voltage increases and approaches a 
critical value $U_{\mathrm{c}}$, two contacting droplets start to coalesce (insert: $1.05 \mathrm{~V}$, Fig 2(a)), which is also indicated by the jump in the current plot. The coalescence of droplets driven by an applied electric field is termed electro-coalescence. ${ }^{21-29,31-33}$
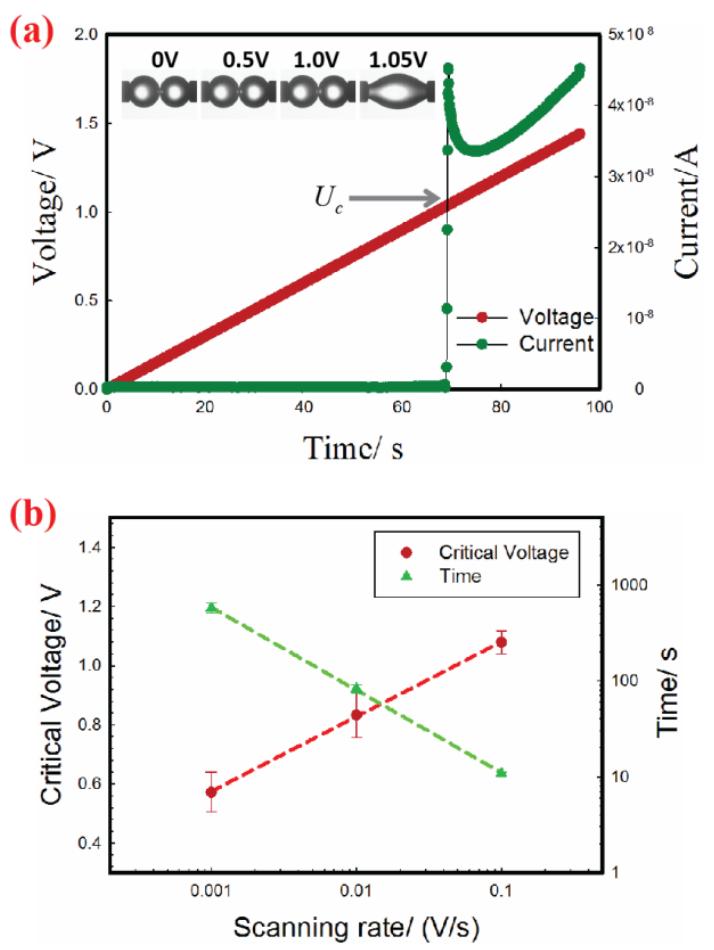

Fig. 2 (a) The applied voltage increases gradually at a scanning rate of $0.015 \mathrm{~V} / \mathrm{s}$ (red dots). When it reaches a critical value $U_{c}$, the droplet pair coalesces, as indicated by the sudden increase of the current (green dots); the insets are microscopic images showing behaviours of the droplet pair under different applied voltage; the outer diameter of the needles is $0.9 \mathrm{~mm}$. (b) Plots of critical voltage (red dots) and time for coalescence to occur (green dots) versus the scanning rate of the applied voltage. For experimental tests in both (a) and (b), the dispersed phase is deionized water and the continuous phase is the paraffin oil with $2 \%$ EM90.

Surprisingly, the value of the critical voltage $U_{\mathrm{c}}$ for droplet electro-coalescence is not constant as reported previously. ${ }^{28,29}$ Instead, $U_{\mathrm{c}}$ changes and depends crucially on the scanning rate of the applied voltage. We systematically test the critical voltages of water droplets stabilized by $2 \%$ EM90 in paraffin oil under an applied voltage with scanning rate ranging from $10^{-3} \mathrm{~V} / \mathrm{s}$ to $10^{-1} \mathrm{~V} / \mathrm{s}$. The results show that, with an increase in scanning rate, the critical voltage increases accordingly (red dots, Fig 2(b)). Moreover, with a faster scanning rate, the time needed for coalescence to occur after the initial application of voltage becomes shorter (green dots, Fig 2(b)). The dependence of critical voltage on scanning rate indicates that the electro-coalescence is a time-dependent dynamic process instead of a simple static problem.

Besides the dependence on the scanning rate, the critical voltage also changes sharply with the surfactant concentration, the nature of the surfactant and even temperature. To confirm the dependence on surfactant concentration, we keep the scanning rate constant $(0.005$ $\mathrm{V} / \mathrm{s}$ ) and measure the critical voltages for three types of surfactant at various concentrations systematically, as shown in Fig 3(a). For each surfactant type, upon an increase in the surfactant concentration, the critical voltage increases significantly initially. However, when the concentration increases above a certain value, the critical voltage reaches a plateau and remains relatively unchanged. Such dependence on concentration is observed for different surfactants including EM 90, Span 80, DC749 and other continuous phases, such as silicone oil, as shown in Fig 3(a). We hypothesize that the specific concentration above which the critical voltage starts to converge has a strong correlation with the critical micelle concentration (CMC) of the surfactants. Surfactants can migrate to interfaces and form a physical barrier to prevent the direct contact of the emulsion droplets. The stability of emulsion is highly dependent on the surface concentration in addition to the properties of surfactants and the fluid phases. The surface concentration of the surfactant is in a dynamic equilibrium with the bulk concentration ${ }^{34-}$ 36. When the bulk concentration of surfactant is above a critical value, the surface concentration becomes saturated and does not increase further. The additional surfactants in the bulk solution will form micelles, instead of migrating to the interfaces; thus, they will not further enhance the stability of emulsion. The concentration where the micelles start to form is termed as critical micelle concentration. We obtain the CMC value of EM90 by measuring the interfacial tension between water and paraffin oil with surfactants at various concentrations (green dotes, Fig 3(b)). From our results, the $\mathrm{CMC}$ is in surprisingly excellent agreement with the threshold concentration above which the critical voltage starts to plateau. With increasing surfactant concentration, the interfacial tension decreases and the critical voltage increases. Both of them start to converge at almost the same value of the concentration. Apart from the surfactant type and concentration, the critical voltage is also highly sensitive to the temperature of the liquid. We systematically test the critical voltages of emulsions stabilized by $2 \%$ Span 80 or $2 \%$ EM90 at temperatures ranging from $23^{\circ} \mathrm{C}$ to $60^{\circ} \mathrm{C}$. The results of both surfactants show that the critical voltage decreases as the temperature increases (Fig 3(c)).
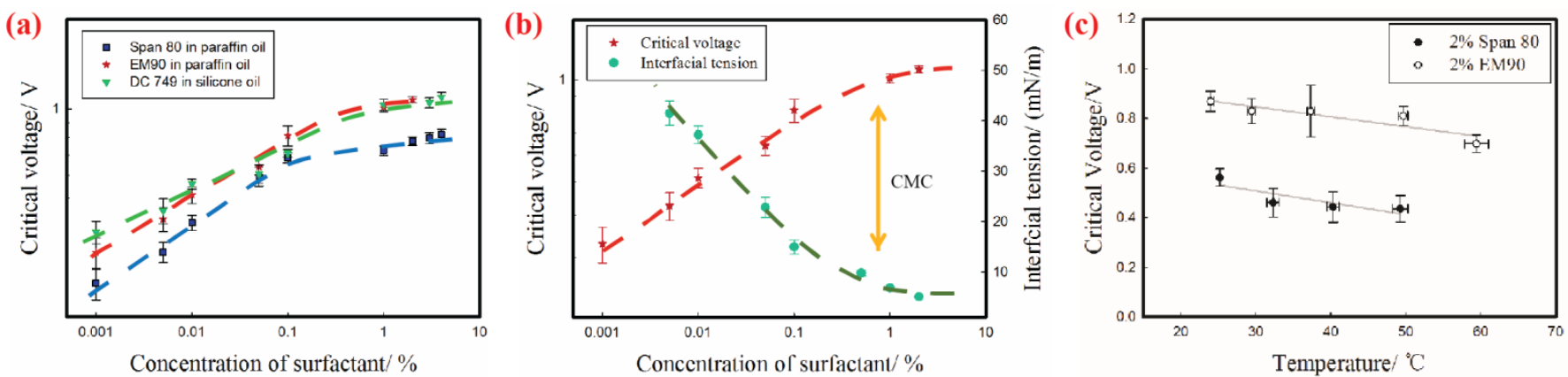
Fig. 3 (a) Plots of critical voltage versus concentration of surfactant. Critical voltages of Span 80 (blue dots), EM90 (red dots) and DC749 (green dots) with concentration range from $0.001 \%$ to $4 \%$ are tested through electro-coalescence in DC voltage. (b) A plot of the interfacial tension and critical voltage as a function of the concentration of a non-ionic surfactant, EM 90, in paraffin oil. (c) Plots of critical voltage versus temperature. Two types of surfactants are tested: $2 \%$ Span 80 (filled dots) and 2\% EM 90 (open dotes); The scanning rate of applied voltage is fixed at $0.005 \mathrm{~V} / \mathrm{s}$.

\section{Electric compression and film thinning dynamics}

To understand the behaviours of emulsion droplets under electric voltage, we first investigate the mechanism of droplet coalescence driven by electric stress. When two emulsion droplets are approaching each other, the opposing interfaces are deformed or flattened due to the existence of a surfactant layer as a physical barrier. A thin lamellar oil film is formed between the two water/oil interfaces, ${ }^{37}$ as shown schematically in Fig 4(a). When an electric voltage is applied to the emulsions, the voltage drop is the most significant across the oil film as the oil is a dielectric fluid being much less conductive $\left(10^{-12} \sim 10^{-10} \mathrm{~S} / \mathrm{cm}\right.$ ) than the aqueous phase (see

(a)

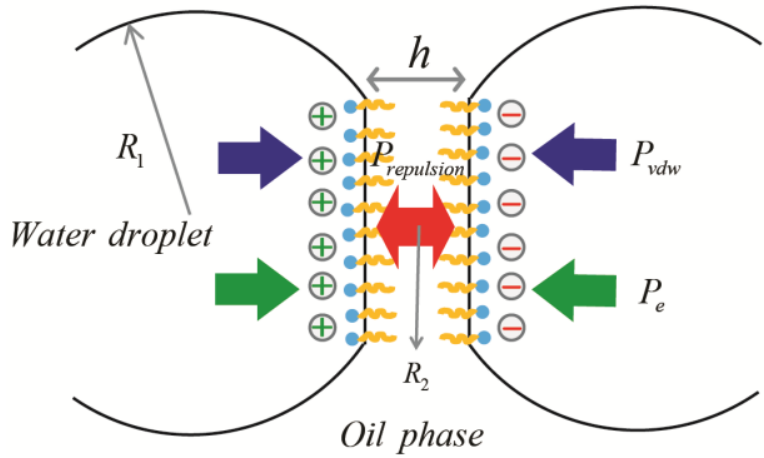

(c)

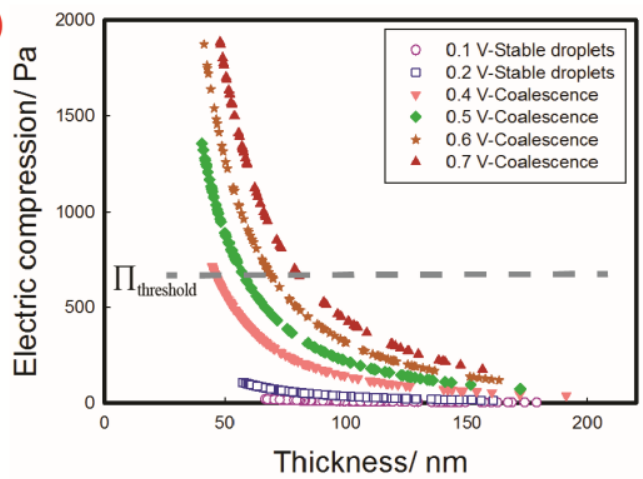

ESI 1). Thus, the thin oil film can be treated as a parallel-plate capacitor charged by the applied voltage $U$. The charge density on the interfaces is estimated as $\sigma=\varepsilon_{\mathrm{r}} \varepsilon_{0} U / h$, where $h$ is the film thickness. These interfacial charges attract the opposite interface and cause an electric compression $P_{\mathrm{e}}$ to the interfaces, which can be calculated by the Maxwell stress ${ }^{38,39} P_{e}=0.5 \cdot \varepsilon_{\mathrm{r}} \varepsilon_{0} \cdot(U / h)^{2}$. In addition, the Laplace pressure $P_{\text {lapalace }}$ also compresses the interfaces, given by Young-Laplace equation: $P_{\text {lapalace }}=2 \gamma / R$, where $R$ is the droplet radius. However, as $R$ is around $1 \mathrm{~mm}$ in our experiment, the Laplace pressure is much smaller in magnitude than the electric compression. We can thus neglect the Laplace pressure effects on the consequent coalescence.

(b)

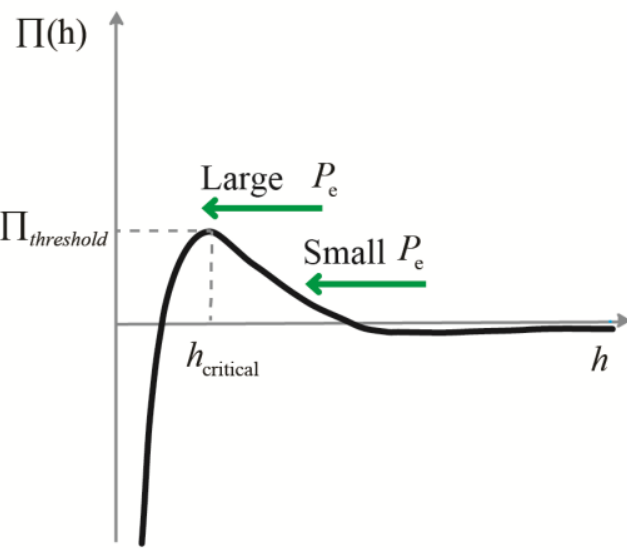

(d)

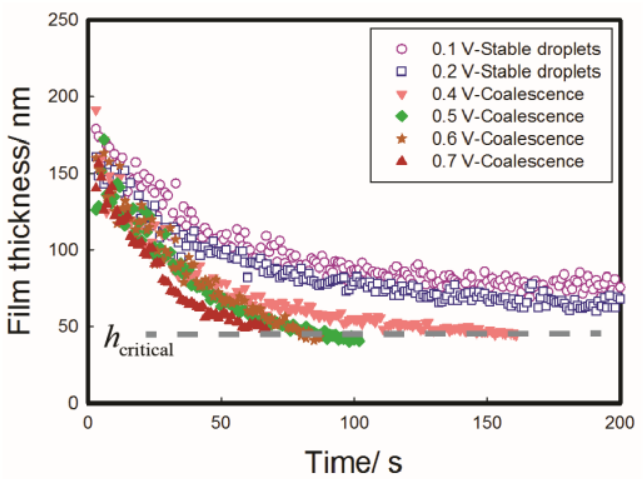

Fig. 4 (a) Schematic illustration of the stresses on the interfaces for oppositely charged emulsion droplets. The electric field induces an electric compression $P_{\mathrm{e}}$ to squeeze the oil film while the disjoining pressure consisting of a repulsive stress $P_{\text {repulsion }}$ and the attractive van der Vaal stress $P_{\mathrm{vdw}}$ prevents the thinning of the gap; $R_{1}$ and $R_{2}$ denote the radii of the droplets and contact area respectively. (b) Disjoining pressure isotherm $\left(\prod_{=} P_{\mathrm{vdw}}+P_{\text {repulsion }}\right)$ versus the film thickness $h$. A metastable point $\left(h_{\text {critical }}\right.$, $\left.\prod_{\text {threshold }}\right)$ exists, indicating the pressure barrier that resists the coalescence of the droplets kinetically. The disjoining pressure barrier can be overcome when $P_{\mathrm{e}}$ exceeds $\prod_{\text {threshold. }}$ (c) A plot of electric compression $P_{\mathrm{e}}$ versus film thickness $h$ at constant voltages: $0.1 \mathrm{~V}$ (purple dots), $0.2 \mathrm{~V}$ (blue dots)), $0.4 \mathrm{~V}$ (pink dots), $0.5 \mathrm{~V}$ (green dots), $0.6 \mathrm{~V}$ (yellow dots) and $0.7 \mathrm{~V}$ (red dots). Coalescence occurs when $P_{\mathrm{e}}$ exceeds the $\prod_{\text {threshold }}$ (dotted line). (d) Film thinning dynamics at different applied voltages. Coalescence occurs when the film thickness is below the critical thickness $h_{\text {critical }}$ (dotted line). For all experiments in plot (c) and (d), the dispersed phase is deionized water and the continuous phase is paraffin oil with $2 \%$ Span 80 .

We attribute the electro-coalescence of emulsions to a sufficiently large electric compression which helps to overcome the threshold disjoining pressure. The electric compression $P_{\mathrm{e}}$, resulting from the oppositely charged interfaces, drains out the oil and leads to the thinning of the film. Meanwhile, the disjoining pressure barrier $\prod_{\text {threshold }}$ opposes the $P_{\mathrm{e}}$ and prevents the film from thinning, as shown schematically in Fig 4(b). With a small applied voltage, $P_{\mathrm{e}}$ is insufficient to overcome the $\prod_{\text {threshold }}$. As a result, the oil film is thinned to a thickness where the electric compression is balanced by the compression and no coalescence will occur. To prove this, we 
directly apply a constant voltage to the emulsion droplets (water droplets dispersed in paraffin oil with $2 \%$ Span 80 ). Indeed, at small voltages $(0.1 \mathrm{~V}, 0.2 \mathrm{~V})$, the oil film maintains a certain thickness without coalescence as shown by the purple and blue dots in Fig $4(\mathrm{c})$. When the applied voltage is sufficiently large $(0.4 \mathrm{~V}-0.7 \mathrm{~V}), P_{\mathrm{e}}$ exceeds $\prod_{\text {threshold }}$ and leads to the coalescence, as shown by the pink, green, yellow and red dots in Fig 4(c).

For coalescence to occur, the oil film needs to be thinned to the critical thickness ${ }^{40-42}$ as indicated by the dotted line in Fig 4(d). Though any sufficiently large voltage can cause electro-coalescence, the time period needed for coalescence to occur after initial voltage application is determined by the film thinning dynamics. The rate of film thinning depends on the exact value of voltage and can be estimated using the Stefan-Reynolds equation, which considers the water/oil interfaces as two parallel plates being pushed towards each other by the stress $\Delta P::^{28,42}$

$$
\frac{d\left(1 / h^{2}\right)}{d t}=\frac{4 \Delta P}{3 \eta r^{2}},
$$

where $\eta$ is the viscosity of the oil, and $r$ is the radius of the contact area. The film thinning dynamics is driven by the electric compression and prevented by the disjoining pressure barrier. Thus, the stress applied on film thinning is: $\Delta P=P_{\mathrm{e}}-\Pi(h)$. With a larger voltage, the electric compression $P_{\mathrm{e}}$ is larger, and hence, the stress $\Delta P$ for film thinning increases accordingly. As a result, the film thinning rate is faster and the time taken for coalescence to occur is shorter. Indeed, the evolution profile of the film thickness agrees well with this prediction, as shown in Fig 4(d). When the applied voltage is $0.4 \mathrm{~V}$, the time takes for film to be thinned to the critical thickness $h_{\text {critical }}$ is around 160s (pink dots, Fig 4(d)). As the applied voltage is increased to $0.7 \mathrm{~V}$, the time is shortened to $70 \mathrm{~s}$ (red dots, Fig 4(d)). Such film thinning dynamics depend on the magnitude of the applied voltage; this may also contribute to the observed dependence of critical voltages on the scanning rate (see ESI 2).

\subsection{Determining threshold disjoining pressure for characterizing the emulsion stability}

Sufficiently large electric compression, caused by applied voltage, can overcome the disjoining pressure barrier and leads to coalescence. Based on this, we propose a method to deduce $\prod_{\text {threshold }}$. To obtain the value of $\prod_{\text {threshold, }}$, we apply a small voltage $U_{\mathrm{c}}$ just enough to trigger coalescence. In doing so, the film is ensured to be gradually thinned to the critical thickness $h_{\mathrm{c}}$ right before coalescence. Thus, the left term of film thinning equation (Eq. 1) at $h_{\mathrm{c}}$ becomes zero: $d\left(1 / h^{2}\right) / d t=0$. As a result, we can estimate the $\prod_{\text {threshold }}$ using the following formula:

$$
\prod_{\text {threshold }}=P_{e}=0.5 \cdot \varepsilon_{r} \varepsilon_{0} \cdot\left(U_{c} / h_{c}\right)^{2} .
$$

To demonstrate our approach, we systematically estimate $\prod_{\text {threshold }}$ for emulsions stabilized by surfactant EM90 or Span 80 at various concentrations, as shown in Fig 5. Indeed, we find a high dependence of $\prod_{\text {threshold }}$ on the concentration for both surfactants, in agreement with a previous study ${ }^{18}$ : as the surfactant concentration increases from zero to the CMC value, the $\prod_{\text {threshold }}$ increases. With a further increase above the CMC value, the $\prod_{\text {threshold }}$ starts to plateau at a maximum value, which may due to the saturation of surfactants on interfaces.

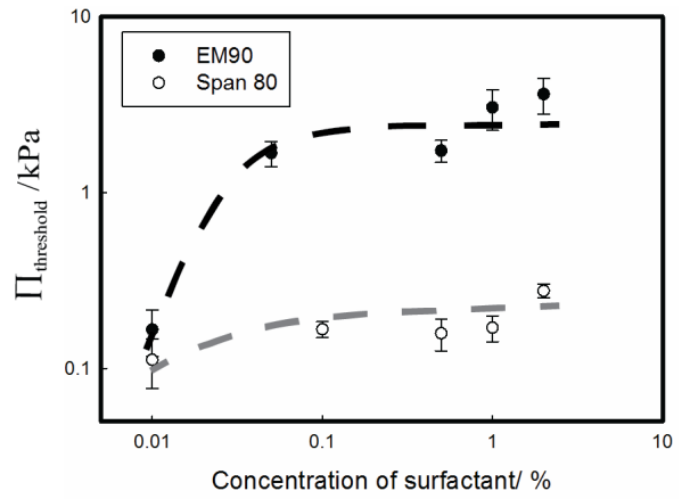

Fig. 5 The threshold disjoining pressure $\prod_{\text {threshold }}$ for emulsions stabilized by EM90 and Span80 at various concentrations.

Moreover, the threshold disjoining pressure $\prod_{\text {threshold }}$ should change with the nature of surfactants. ${ }^{11,16}$ Indeed, the threshold disjoining pressure values measured by the proposed technique for EM90 and Span 80 differs from each other significantly, as shown in Fig 5. The difference in the value of $\prod_{\text {threshold }}$ should be reflected by a corresponding difference in the stability of the emulsions. To confirm this, two common methods, namely centrifugationaccelerated compression and thermal heating, are applied to characterize the stability of emulsions stabilized by $2 \%$ EM90 or $2 \%$ Span 80. After centrifugation, the emulsion layer of the $2 \%$ EM90 sample is much thicker than that of the $2 \%$ Span 80 sample at rotation speeds of $1000 \mathrm{rpm}$ and $2000 \mathrm{rpm}$ (Fig 6(a)), suggesting that the emulsion stabilized by EM90 is more robust against coalescence than that by Span 80 . Therefore, the $\prod_{\text {threshold }}$ of $2 \%$ EM90-stabilized emulsions should be higher than that of the $2 \%$ Span 80 counterpart, in agreement with the results characterized by our approach. In addition, in our thermal heating experiments, after incubation at $50^{\circ} \mathrm{C}$ for 65 hours, the $2 \%$ EM 90 -stabilized emulsion drops are still uniform in size, while the $2 \%$ Span 80 -stabilized emulsion drops coalesce rapidly and subsequently show significant polydispersity, as shown in Fig 6(b). This also suggests that 2\% EM90 results in a higher $\prod_{\text {threshold }}$ than $2 \%$ Span 80 does; this again matches our results. Thus, our approach based on the electro-coalescence of droplets can precisely and accurately measure the threshold disjoining pressure which helps predict the emulsion stability quantitatively. (a)

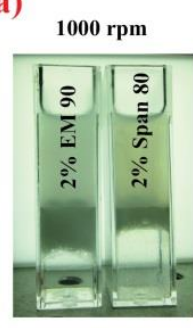

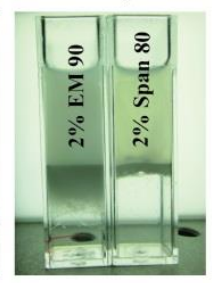

$2000 \mathrm{rpm}$ (b)

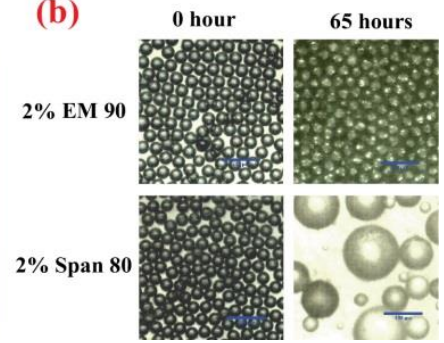

Fig. 6 (a) Centrifugal acceleration to compare the performance of $2 \%$ EM90 and $2 \%$ Span80 on stabilizing water emulsions in paraffin oil. The $2 \%$ EM90-stabilized emulsions are more stable as they have a thicker emulsion layer than that of 2\% Span80 at rotation speeds of $1000 \mathrm{rpm}$ and $2000 \mathrm{rpm}$. (b) Microscopic images demonstrating emulsion stability under thermal heating. Uniform water droplets dispersed in paraffin oil with surfactants are put in an oven at $50^{\circ} \mathrm{C}$. After 65 hours, $2 \%$ EM90 stabilized emulsions remain stable while $2 \%$ Span 80 stabilized ones not; Scale bars are 400 microns. 


\section{Conclusions}

We have investigated the droplet-based electro-coalescence of emulsion droplets using our custom-built device systematically. Interestingly, the critical voltage upon which electro-coalescence occurs is highly dependent on the scanning rate of the applied DC voltage, surfactant type and concentration, and even temperature. We interpret that these factors change the critical voltage by affecting the disjoining pressure and the corresponding film thinning dynamics. Based on these understandings, we have devised an approach to quantitatively characterize the stability of emulsions using the threshold disjoining pressure obtained by electrocoalescence. The reliability of this approach is further proved by corroborating with results of centrifuge-accelerated compression and thermal heating. We believe the simplicity and reliability of our approach facilitates its use in the design of emulsion-based formulations.

\section{Acknowledgements}

We thank Dr. Tiantian Kong and Yang Song for the helpful discussion and comments. This research was supported by the Early Career Scheme (HKU 707712P) and the General Research Fund (HKU 719813E and 17304514) from the Research Grants Council of Hong Kong, the General Program (21476189/ B060201) and Young Scholar's Program (NSFC51206138/E0605) from the National Natural Science Foundation of China, as well as the Seed Funding Programme for Basic Research (201211159090, 201311159105) and for Applied Research (201309160035) from the University of Hong Kong.

\section{Notes and references}

To whom the correspondence should be addressed. Email: ashum@hku.hk

${ }^{a}$ Department of Mechanical Engineering, Haking Wong Building, The University of Hong Kong, Hong Kong.

${ }^{b}$ HKU-Shenzhen Institute of Research and Innovation (HKU-SIRI), Shenzhen, Guang Dong, China.

${ }^{c}$ Department of Electrical and Electronic Engineering, Chow Yei Ching Building, The University of Hong Kong, Hong Kong.

$\dagger$ Electronic Supplementary Information (ESI) available: Voltage drop across the oil film. See DOI: 10.1039/b000000x/

1. K. J. Lissant, Emulsions and emulsion technology, Dekker, New York, 1974.

2. D. J. McClements, Critical Reviews in Food Science and Nutrition, 2007, 47, 611-649.

3. C. Gallegos and J. M. Franco, Current Opinion in Colloid \& Interface Science, 1999, 4, 288-293.

4. V. Srinivasan, V. K. Pamula and R. B. Fair, Lab on a Chip, 2004, 4,310-315.

5. C. F. Carlborg, K. B. Gylfason, A. Kaźmierczak, F. Dortu, M. J. B. Polo, A. M. Catala, G. M. Kresbach, H. Sohlström, T. Moh and L. Vivien, Lab on a Chip, 2010, 10, 281-290.

6. M. Kim, M. Pan, Y. Gai, S. Pang, C. Han, C. Yang and S. K. Y. Tang, Lab on a Chip, 2015, DOI: 10.1039/c4lc01465k.

7. S.-H. Kim, J. W. Kim, J.-C. Cho and D. A. Weitz, Lab on a Chip, 2011, 11, 3162-3166.

8. F. Tu and D. Lee, Chemical Communications, 2014, 50, 15549-15552. 9. Y. Song, A. Sauret and H. C. Shum, Biomicrofluidics, 2013,7,061301. 10. S. S. Datta, A. Abbaspourrad, E. Amstad, J. Fan, S. H. Kim, M. Romanowsky, H. C. Shum, B. Sun, A. S. Utada and M. Windbergs, Advanced Materials, 2014, 26, 2205-2218.

11. S. Cosima and K. Regine von, Journal of Physics: Condensed Matter, 2003, 15, R1197.

12. B. V. Derjaguin and N. V. Churaev, Journal of Colloid and Interface Science, 1974, 49, 249-255.
13. A. Atta, D. G. Crawford, C. R. Koch and S. Bhattacharjee, Langmuir, 2011, 27, 12472-12485.

14. R. Verma, A. Sharma, I. Banerjee and K. Kargupta, Journal of Colloid and Interface Science, 2006, 296, 220-232.

15. S. Herminghaus, Physical Review Letters, 1999, 83, 2359-2361.

16. B. V. Derjaguin, N. V. Churaev and V. M. Muller, in Surface Forces, Springer US, 1987, DOI: 10.1007/978-1-4757-6639-4_2, ch. 2, pp. 25-52. 17. B. V. Derjaguin and N. V. Churaev, Journal of Colloid and Interface Science, 1978, 66, 389-398

18. J. Bibette, D. C. Morse, T. A. Witten and D. A. Weitz, Physical Review Letters, 1992, 69, 2439-2442.

19. S. H. Kim, H. Hwang, C. H. Lim, J. W. Shim and S. M. Yang, Advanced Functional Materials, 2011, 21, 1608-1615.

20. H. Chen, J. Li, H. C. Shum, H. A. Stone and D. A. Weitz, Soft Matter, 2011, 7, 2345-2347.

21. J. H. Kim, J.-H. Choi, J. Y. Sim, W. C. Jeong, S.-M. Yang and S.-H. Kim, Langmuir, 2014, 30, 5404-5411.

22. T. Brugarolas, F. Tu and D. Lee, Soft Matter, 2013, 9, 9046-9058.

23. L. Rosenfeld, L. Fan, Y. Chen, R. Swoboda and S. K. Y. Tang, Soft Matter, 2014, 10, 421-430.

24. M. N. Jones, K. J. Mysels and P. C. Scholten, Transactions of the Faraday Society, 1966, 62, 1336-1348.

25. S. Tcholakova, N. D. Denkov, I. B. Ivanov and B. Campbell, Langmuir, 2002, 18, 8960-8971.

26. H. Feng, D. Ershov, T. Krebs, K. Schroen, M. A. C. Stuart, J. van der Gucht and J. Sprakel, Lab on a Chip, 2015, 15, 188-194.

27. J. Li, Y. Wang, H. Chen and J. Wan, Lab on a Chip, 2014, 14, 43344337.

28. X. Niu, F. Gielen, A. J. deMello and J. B. Edel, Analytical Chemistry, 2009,81,7321-7325.

29. C. Priest, S. Herminghaus and R. Seemann, Applied Physics Letters, $2006,89,134101$

30. R. K. Shah, H. C. Shum, A. C. Rowat, D. Lee, J. J. Agresti, A. S. Utada, L.-Y. Chu, J.-W. Kim, A. Fernandez-Nieves, C. J. Martinez and D. A. Weitz, Materials Today, 2008, 11, 18-27.

31. L. Mazutis and A. D. Griffiths, Lab on a Chip, 2012, 12, 1800-1806. 32. J. G. Kralj, M. A. Schmidt and K. F. Jensen, Lab on a Chip, 2005, 5, 531-535.

33. L. Mazutis, J.-C. Baret and A. D. Griffiths, Lab on a Chip, 2009, 9, 2665-2672.

34. A. D. McNaught, Compendium of chemical terminology, Blackwell Science Oxford, 1997.

35. A. Dominguez, A. Fernandez, N. Gonzalez, E. Iglesias and L. Montenegro, Journal of Chemical Education, 1997, 74, 1227.

36. J. N. Phillips, Trans. Faraday Soc., 1955, 51, 561-569.

37. C. Stubenrauch and R. von Klitzing, Journal of Physics: Condensed Matter, 2003, 15, R1197.

38. M. R. Anklam, D. A. Saville and R. K. Prud'Homme, Langmuir, 1999, 15, 7299-7307.

39. F. Mostowfi, J. Czarnecki, J. Masliyah and S. Bhattacharjee, Journal of Colloid and Interface Science, 2008, 317, 593-603.

40. E. D. Manev and J. K. Angarska, Colloids and Surfaces A: Physicochemical and Engineering Aspects, 2005, 263, 250-257.

41. W. A. B. Donners and A. Vrij, Colloid and Polymer Science, 1978, 256, 804-813.

42. E. D. Manev and A. V. Nguyen, Advances in Colloid and Interface Science, 2005, 114-115, 133-146.

43. B. Steinhaus, P. T. Spicer and A. Q. Shen, Langmuir, 2006, 22, 53085313. 